А.А. Кралин, Е.В. Крюков, В.В. Еременко

Нижегородский государственный технический университет

им. Р.Е. Алексеева, Нижний Новгород, Россия

ПРИМЕНЕНИЕ ТИРИСТОРНОГО РЕГУЛЯТОРА

ВОЛЬТОДОБАВОЧНОГО НАПРЯЖЕНИЯ В РАДИАЛЬНЫХ

РАСПРЕДЕЛИТЕЛЬНЫХ ЭЛЕКТРИЧЕСКИХ СЕТЯХ 6-10 КВ ДЛЯ РЕГУЛИРОВАНИЯ ПОТОКОВ МОЩНОСТИ

\footnotetext{
Переход к интеллектуальным энергосистемам является ключевой задачей российской и зарубежной энергетической индустрии. Одним из параметров интеллектуализации является способность управлять потоками мощности в электрических сетях. Это возможно реализовать с помощью устройств, позволяющих контролировать параметры и режимы работы электрической сети. Такое устройство разработано в Нижегородском государственном техническом университете им. Р.Е. Алексеева. Статья посвящена исследованию эффективных областей работы тиристорного регулятора вольтодобавочного напряжения (ТРВДН) в параллельно работающих линиях электропередачи (ЛЭП), а также определению зависимости КПД системы, токовых и мощностных характеристик от значения вольтодобавочного напряжения. Наиболее удобным средством решения поставленных задач является моделирование в программном комплексе MatLab Simulink, обладающем высоким быстродействием и необходимой точностью измерений. Разработанная имитационная модель ТРВДН позволяет реализовать продольное, поперечное и продольно-поперечное регулирование напряжения. Внедрение этой модели в исследуемый участок распределительной электрической сети (РЭС) позволило провести исследования регулирования потоков мощности с помощью разработанного устройства. Была определена область стабилизации токов в параллельно работающих воздушной и кабельной линиях. Работа устройства в данной области позволяет увеличить пропускную способность ЛЭП и повысить КПД системы в целом. В программном комплексе Mathcad была разработана математическая модель ТРВДН. С помощью метода узловых потенциалов были получены зависимости различных параметров РЭС исследуемого участка. Анализ характеристик показал, что внедрение устройства в радиальную РЭС с параллельно работающими ЛЭП повысит эффективность передачи электроэнергии потребителям.

Ключевые слова: тиристорный регулятор вольтодобавочного напряжения, распределительная электрическая сеть, потокораспредление, неоптимальная загрузка ЛЭП, пропускная способность, имитационная модель.
} 


\author{
A.A. Kralin, E.V. Kryukov, V.V. Eremenko \\ Nizhny Novgorod State Technical University named after R.E. Alekseeva, \\ Nizhny Novgorod, Russian Federation

\section{THYRISTOR VOLTAGE REGULATOR APPLICATION IN RADIAL ELECTRICAL NETWORKS 6-10 kV FOR POWER FLOW CONTROL}

The transition to intellectual electrical networks is a crucial task of the Russian and foreign energy industry. One of the parameters of intellectualization is the ability to control the flow of power in electrical networks. It is possible to implement it with the help of devices that allow controlling the parameters and operation modes of the electrical network. This device was developed at the Nizhny Novgorod State Technical University. R.E. Alekseeva. The article is devoted to the study of the effective areas of the thyristor voltage regulator (TVR) operation in parallel operating power transmission lines (PTL), as well as to determine the dependence of efficiency factor, current and power characteristics on the value of the booster voltage. The most convenient way to solve the problems is modeling in the MatLab Simulink software package, which has high speed and the required measurement accuracy. The developed simulation TVR model allows to implement longitudinal, transverse and longitudinaltransverse voltage regulation. The introduction of this model into the studied area of the distribution electric network (DEN) allowed to conduct investigation of the power flows regulation with the application of the developed device. The area of current stabilization in parallel operating air and cable lines was determined. The operation of the device in this area allows to increase the transmission capacity of power lines and increase the network efficiency factor as a whole. In the Mathcad software package, a mathematical model of the TVR was developed. Using the method of nodal potentials, the dependences of various parameters of the DEN of the studied area were obtained. The analysis of the characteristics has shown that the introduction of the device into the radial DEN with parallel operating power lines will increase the efficiency of power transmission to consumers.

Keywords: thyristor voltage regulator, distribution electrical network, power flow, non-optimal power line load, transmission capacity, simulation model.

Введение. Одной из важнейших проблем электросетевого комплекса России является повышение эффективности использования существующих линий электропередач (ЛЭП) всех классов напряжения за счет увеличения их пропускной способности. Регулирование потоков мощности с использованием устройств продольно-поперечного регулирования напряжения позволяет оптимизировать загрузку ЛЭП, повысить их надежность и управляемость [1-3]. В настоящее время основное внимание уделено исследованию режимов работы высоковольтных ЛЭП. Вместе с тем проблемы функционирования распределительных электрических сетей (РЭС) среднего напряжения с использованием устройств продольной, поперечной и продольно-поперечной компенсации на основе трансформаторно-тиристорных регуляторов напряжения исследованы недостаточно [4-6]. 
Предложенный авторами тиристорный регулятор вольтодобавочного напряжения (ТРВДН) позволяет регулировать как величину, так и угол сдвига основной гармоники выходного напряжения. Использование разработанного устройства в сетях среднего напряжения различной конфигурации позволяет управлять потоками активной и реактивной мощностей, а также стабилизировать напряжение в пределах, установленных ГОСТ [7-9].

С целью определения зон работы ТРВДН, характеризующихся увеличением пропускной способности РЭС, обладающих высоким КПД, предельно допустимыми токовыми нагрузками, необходимо определить регулировочные характеристики устройства.

Цели данной работы - исследование режимов работы РЭС совместно с ТРВДН при различных параметрах распределительной сети, определение зон эффективного регулирования потоков мощности.

1. Схемотехнические решения. Предложенное авторами схемотехническое решение силовой части ТРВДН (рис. 1) содержит шунтовой трехфазный трансформатор с модулями продольного и поперечного регулирования и три сериесных трансформатора.

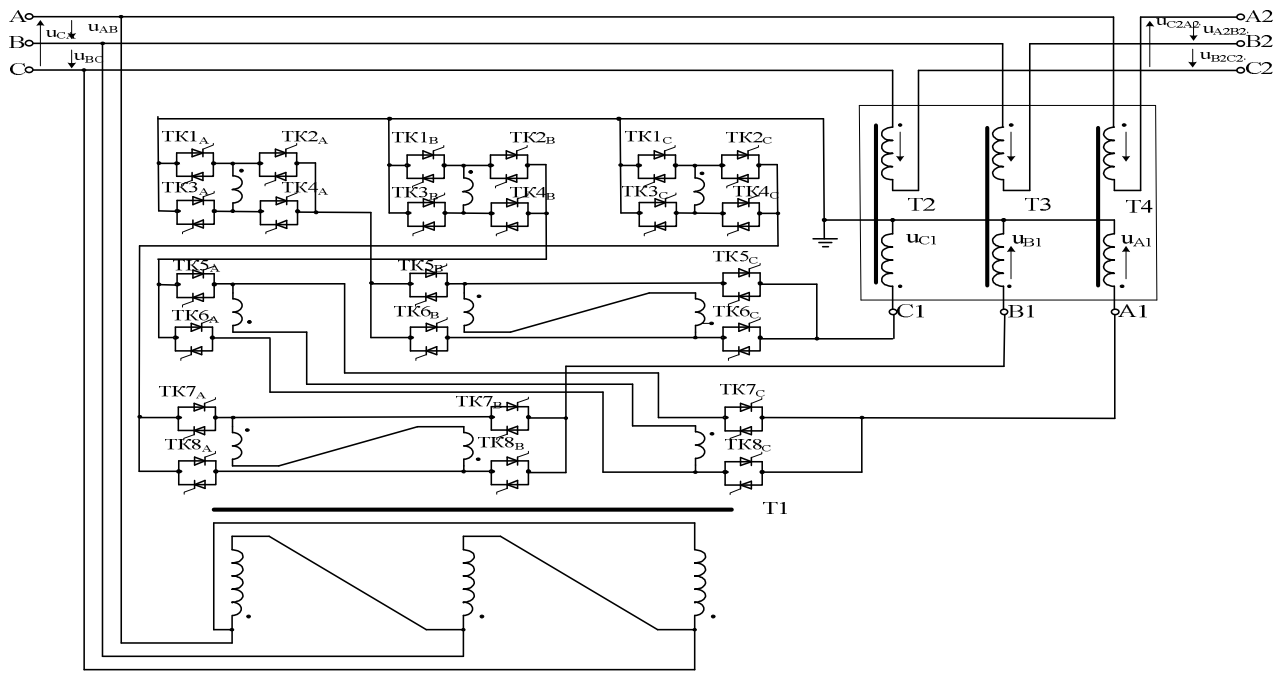

Рис. 1. Силовая схема ТРВДН

Технические характеристики разработанного устройства приведены в табл. 1. Модуль поперечного регулирования осуществляет сдвиг по фазе выходных линейных напряжений относительно входных, 
модуль продольного регулирования управляет величиной выходного напряжения [10]. Регулирование параметров электроэнергии происходит за счет совместной работы этих модулей.

\section{Таблица 1}

Технические характеристики ТРВДН

\begin{tabular}{|c|c|}
\hline $\begin{array}{c}\text { Угол сдвига основной гармоники выходного } \\
\text { напряжения относительно входного }\end{array}$ & $\theta= \pm 5^{\circ}$ \\
\hline $\begin{array}{c}\text { Дискретность изменения сдвига основной гармоники } \\
\text { выходного напряжения }\end{array}$ & $\Delta \theta \leq 1,5 \%$ \\
\hline $\begin{array}{c}\text { Диапазон регулирования величины выходного } \\
\text { напряжения относительно входного }\end{array}$ & $D= \pm 10 \%$ \\
\hline $\begin{array}{c}\text { Дискретность регулирования действующего значения } \\
\text { выходного напряжения }\end{array}$ & $\Delta D \leq 1,5 \%$ \\
\hline Мощность нагрузки & $S_{\mathrm{H}} \leq 1000$ кВА \\
\hline
\end{tabular}

Первичные обмотки шунтового трансформатора Т1 подключены к распределительной сети среднего напряжения. На базе вторичных обмоток реализованы модули продольного и поперечного регулирования трех фаз. Данные модули коммутируются тиристорными ключами ТК1-ТК8. С помощью сериесных трансформаторов, подключенных последовательно в РЭС, вводится продольно-поперечная составляющая напряжения, управляемая тиристорами [11, 12]. Наиболее удобным средством исследования электромагнитных процессов ТРВДН является имитационное моделирование.

3. Имитационное моделирование РЭС с ТРВДН. Имитационная модель радиальной электрической сети с ТРВДН разработана в программном комплексе MatLab/Simulink [13-16]. В качестве объекта моделирования выбран участок РЭС 6 кВ в виде параллельно работающих воздушной и кабельной линий, по которым осуществляется передача электрической мощности от генератора энергосистемы. Однолинейная схема сети приведена на рис. 2. ТРВДН размещен в конце воздушной ЛЭП.

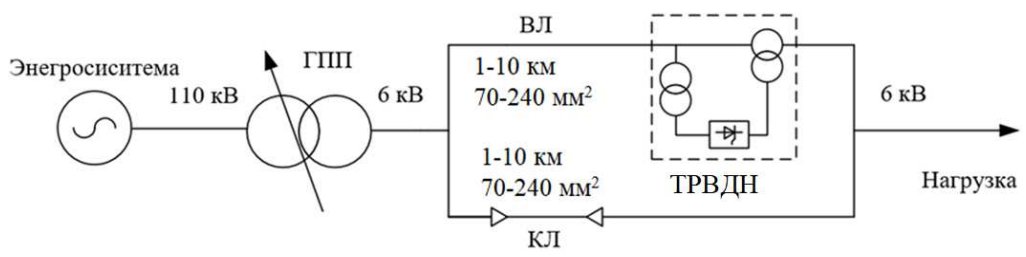

Рис. 2. Однолинейная схема радиальной распределительной сети с ТРВДН 
Имитационная модель рассматриваемой РЭС с ТРВДН представлена на рис. 3.

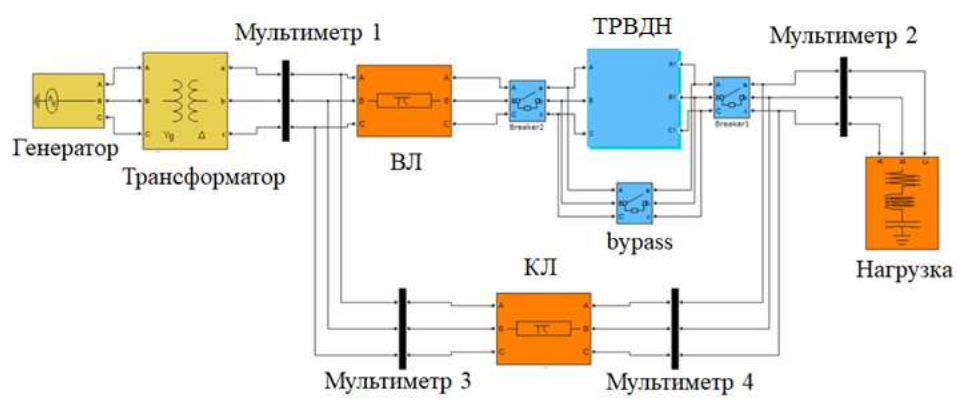

Рис. 3. Имитационная модель исследуемого участка сети с ТРВДН

Модель состоит из 7 основных блоков: 1 - генератор - смоделирован идеальным трехфазным источником питания; 2 - трансформатор представлен понижающим трехфазным двухобмоточным трансформатором 110/6 кB; 3 -линия L1 - кабельная ЛЭП; 4 - линия L2 - воздушная ЛЭП; 5 - высоковольтная нагрузка мощностью 1 MВА представлена активно-индуктивным сопротивлением; 6 - измерительные приборы позволяют снимать значения токов, напряжения, активных и реактивных мощностей; 7 - имитационная модель блока ТРВДН (рис. 4).

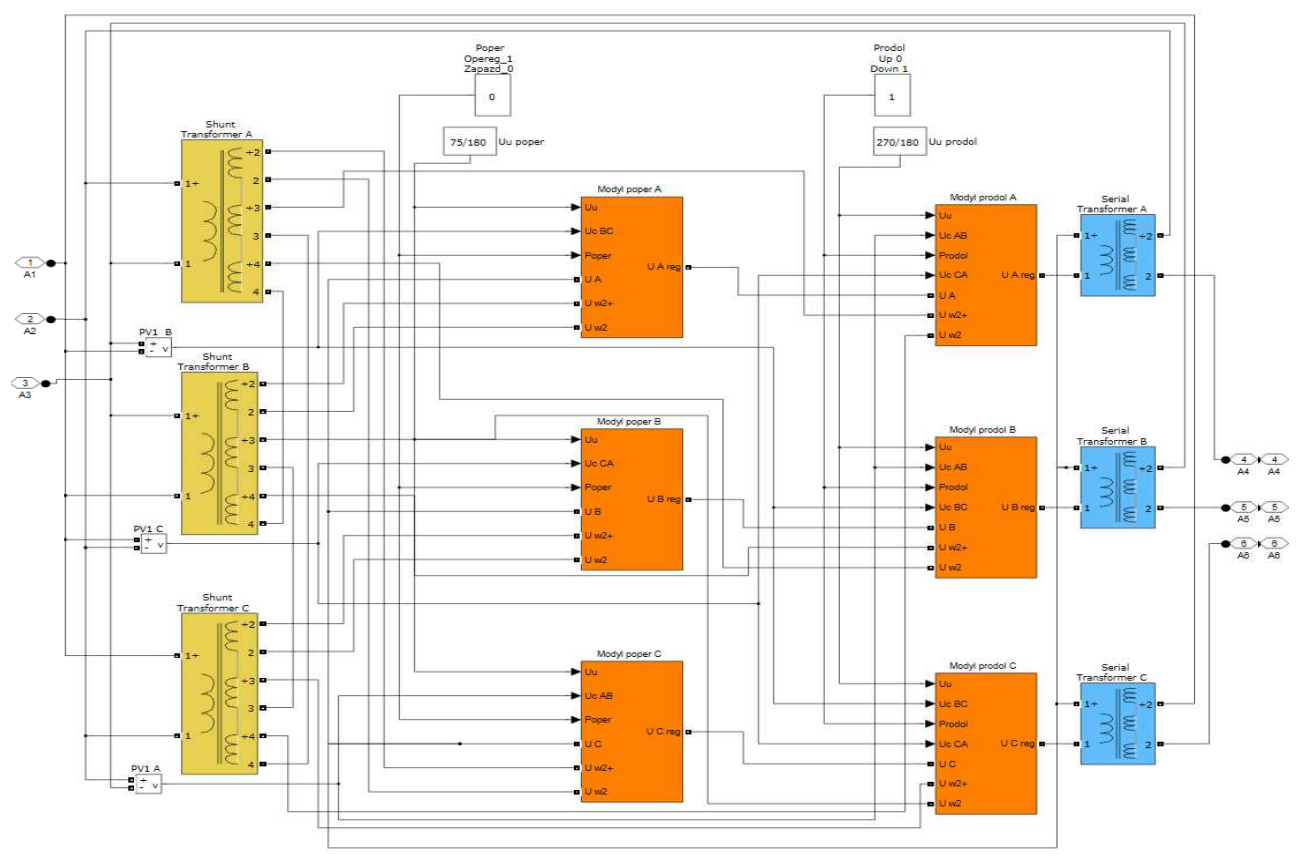

Рис. 4. Имитационная модель блока ТРВДН 
Параметры элементов распределительной электрической сети, используемые для реализации имитационной модели, представлены в табл. 2.

\section{Таблица 2}

Параметры элементов сети

\begin{tabular}{|c|c|c|c|c|c|}
\hline $\begin{array}{c}\text { Наименование } \\
\text { элемента }\end{array}$ & $U$, кB & $\varphi^{\circ}$ & $R$, Ом & $X$, Ом & $l$, км \\
\hline Источник & 110 & 120 & $1 \mathrm{E}-10$ & 0 & - \\
\hline Трансформатор & $110 / 6$ & - & 7,95 & 139 & - \\
\hline Кабельная линия L1 & - & - & $0,129-0,443$ & $0,071-0,08$ & $1-10$ \\
\hline Воздушная линия L2 & - & - & $0,132-0,46$ & $0,071-0,08$ & $1-10$ \\
\hline ТРВДН & - & - & 0,01 & 0,05 & - \\
\hline Нагрузка & - & - & $6-12$ & $0-10,4$ & - \\
\hline
\end{tabular}

В городских РЭС расстояния между ГПП и потребителем не превышают, как правило, 10 км, поэтому граничные условия длин линий при моделировании составляли 1-10 км [17]. При естественном потокораспределении большая часть тока нагрузки протекает по линии, имеющей меньшее сопротивление. Это может быть обусловлено меньшей длиной или большим сечением ЛЭП $[18,19]$.

Исследования были проведены для анализа изменения токов, распределения потоков мощностей, КПД передачи для двух параллельных линий при изменении величины напряжения модулей продольного и поперечного регулирования ТРВДН. Моделирование проводилось для активной и активно-индуктивной нагрузки при различной длине и сечении линий.

В результате исследований получены параметры параллельных ЛЭП (соотношение их сечений и длин), при которых использование ТРВДН позволяет загрузить линии пропорционально их пропускной способности (рис. 5). Значения токов при этом не превышают длительно допустимых токовых нагрузок для соответствующей линии. Цифрой 1 обозначена область параллельно работающих ЛЭП при естественном потокораспределении (без ТРВДН); область 2 соответствует включению ТРВДН в режиме продольно-поперечного регулирования при активной нагрузке; область 3 - при активно-индуктивной нагрузке. В качестве примера в табл. 3 представлены численные значения электрических величин, полученные в результате проведенного исследования при следую- 
щих параметрах линий: отношение неоднородностей линий $\varepsilon 1 / \varepsilon 2=1,32$; Iv1 = 214 A - длительно допустимый ток кабельной линии при сечении

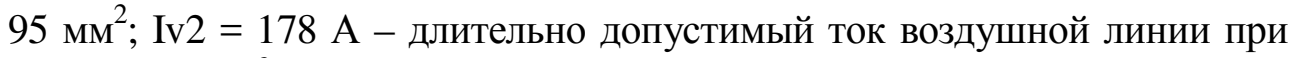

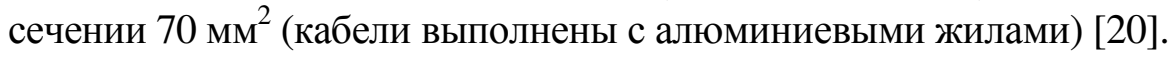

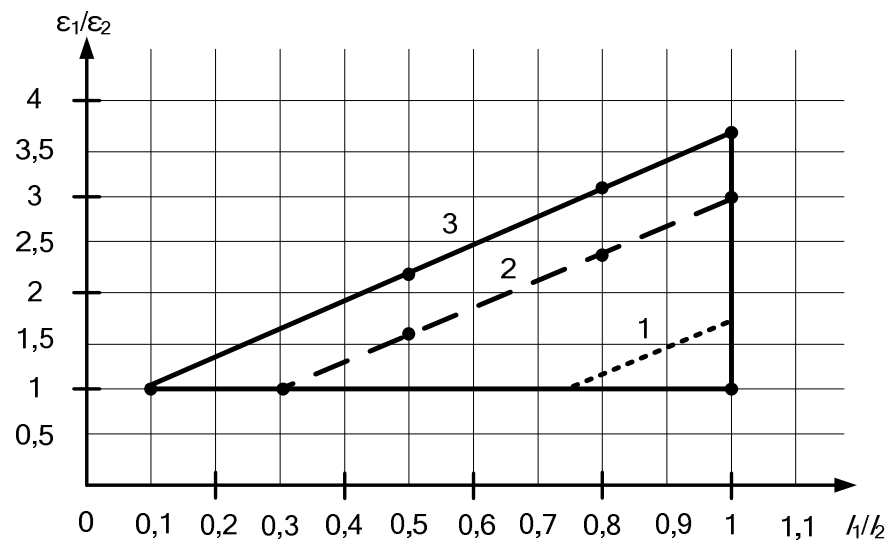

Рис. 5. Области эффективной работы ТРВДН: 1 - без ТРВДН;

$2-$ с ТРВДН при $\varphi=0^{\circ} ; 3-$ с ТРВДН при $\varphi=60^{\circ}$

В качестве базисного напряжения принято входное линейное напряжение UЛ, в качестве базисных токов - длительно допустимые токи линии Iv1 и Iv2, При этом базисная мощность составляет $P_{\text {н }}=1$ MBA. Таким образом, UТРВДН $*$ UТРВДН/Uл, I1* = I1/Iv1, I2* = I2/Iv2, $P_{\mathrm{H}}^{*}=P / P_{\mathrm{H}}$.

Таблица 3

Результаты исследований токов в ЛЭП

\begin{tabular}{|c|c|c|c|c|c|c|c|}
\hline \multirow[t]{2}{*}{$11 / 12$} & \multicolumn{3}{|c|}{$\begin{array}{c}\text { Естественное } \\
\text { потокораспределение } \\
\text { (ТРВДН выключен) }\end{array}$} & \multirow[t]{2}{*}{ UТРВДН* } & \multicolumn{3}{|c|}{$\begin{array}{c}\text { Продольное регулирование } \\
\text { напряжения }\end{array}$} \\
\hline & I1* & $\mathrm{I} 2^{*}$ & $\mathrm{PH}^{*}$ & & I1 ${ }^{*}$ & I2* & $\mathrm{PH}^{*}$ \\
\hline \multicolumn{8}{|c|}{$\varphi=0 \mathrm{o}$} \\
\hline 0,2 & 0,69 & 0,12 & 0,945 & 0,0695 & 0,55 & 0,55 & 0,97 \\
\hline 0,4 & 0,6 & 0,22 & 0,907 & 0,069 & 0,48 & 0,48 & 0,946 \\
\hline 0,6 & 0,52 & 0,28 & 0,878 & 0,0648 & 0,42 & 0,42 & 0,925 \\
\hline 0,8 & 0,46 & 0,32 & 0,855 & 0,0253 & 0,42 & 0,42 & 0,875 \\
\hline 1 & 0,42 & 0,38 & 0,835 & 0,012 & 0,41 & 0,41 & 0,843 \\
\hline \multicolumn{8}{|c|}{$\varphi=60 \mathrm{o}$} \\
\hline 0,2 & 0,7 & 0,12 & 0,48 & 0,048 & 0,44 & 0,44 & 0,49 \\
\hline 0,4 & 0,60 & 0,21 & 0,465 & 0,038 & 0,43 & 0,43 & 0,475 \\
\hline 0,6 & 0,53 & 0,29 & 0,455 & 0,027 & 0,42 & 0,42 & 0,467 \\
\hline 0,8 & 0,49 & 0,34 & 0,447 & 0,016 & 0,42 & 0,42 & 0,452 \\
\hline 1 & 0,42 & 0,39 & 0,44 & 0,007 & 0,41 & 0,41 & 0,441 \\
\hline
\end{tabular}


4. Математическое моделирование РЭС с ТРВДН. Расчет энергетических характеристик при работе ТРВДН в радиальной РЭС с параллельно работающими ЛЭП был реализован в программном комплексе Mathcad. С помощью метода узловых потенциалов были рассчитаны значения токов, напряжений и мощностей исследуемого участка, схема замещения которого представлена на рис. 6. Следует отметить, что при незначительных длинах линий РЭС и напряжениях сети 6-20 кВ кабельную или воздушную линию можно представить активно-индуктивным сопротивлением.

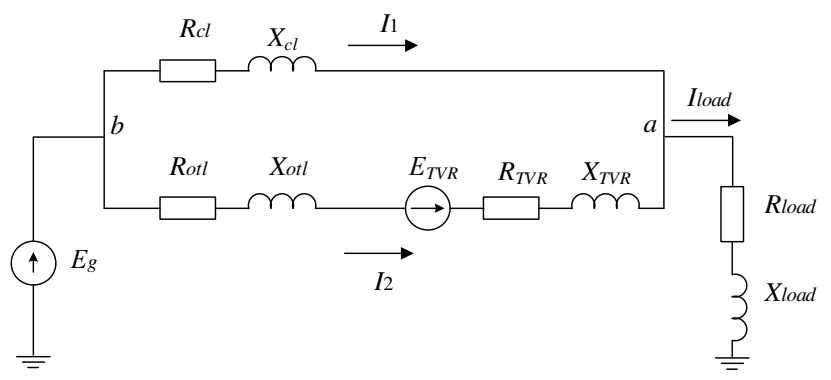

Рис. 6. Схема замещения РЭС с ТРВДН.

С учетом принятых допущений были составлены уравнения по методу узловых потенциалов, решение которых позволяет определить необходимые электромагнитные параметры системы:

$$
\begin{gathered}
\varphi_{b a}=\frac{\left(\frac{U_{\Gamma}}{Z_{\text {нагр }}}-\frac{U_{\text {тРвдн }}}{Z_{\text {вл }}+Z_{\text {тРвдн }}}\right)}{\left(\frac{1}{Z_{\text {кл }}}+\frac{1}{Z_{\text {вл }}+Z_{\text {тРВдн }}}+\frac{1}{Z_{\text {нагр }}}\right)} \\
I_{1}=\frac{\varphi_{b a}}{Z_{\text {кл }}} \\
I_{2}=\frac{\varphi_{b a}+U_{\text {ТРВДН }}}{Z_{\text {вл }}+Z_{\text {ТРВДН }}} \\
I_{\text {нагр }}=-\frac{\varphi_{b a}-U_{\Gamma}}{Z_{\text {нагр }}},
\end{gathered}
$$

где $I_{1}$ - ток в кабельной линии; $I_{2}$ - ток в воздушной линии; $I_{\text {нагр }}$ - ток нагрузки; $Z_{\text {кл }}$ полное сопротивление кабельной линии; $Z_{\text {вл }}-$ полное сопротивление воздушной линии; $Z_{\text {нагр }}$ - полное сопротивление нагрузки; $U_{\text {г }}$ 
ЭДС генератора, $U_{\text {ТРВдн }}$ - поперечная составляющая напряжения ТРВДН, $\varphi_{b a}-$ напряжение между двумя потенциалами точек $b$ и $a$.

Расчеты проводились при изменении продольной, поперечной и продольно-поперечной составляющей напряжения ТРВДН с шагом дискретизации $0,04 U_{\text {Tрвдн }}{ }^{*}$. На рис. 7 и 8 в качестве примера приведены зависимости КПД передачи, токов и мощности нагрузки при продольно-поперечном регулировании напряжения для линий с параметрами $\varepsilon_{1} / \varepsilon_{2}=1,32 ; l_{1} / l_{2}=0,2$.

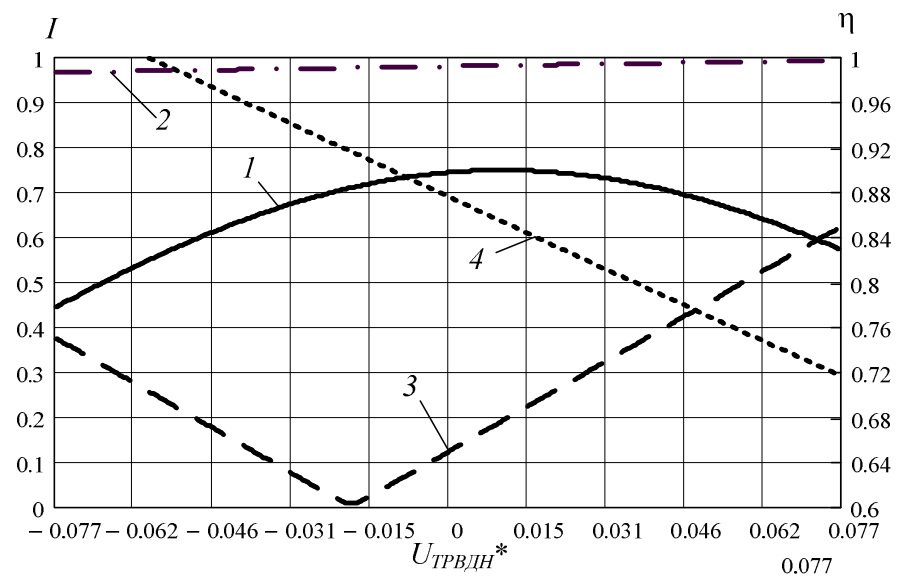

Рис. 7. Зависимости токов нагрузки линий, КПД системы при продольно-поперечном регулировании напряжения ТРВДН для угла нагрузки $\varphi=60^{\circ}: 1-\eta, 2-I_{\text {нагр* }}, 3-I_{2^{*}}, 4-I_{1^{*}}$

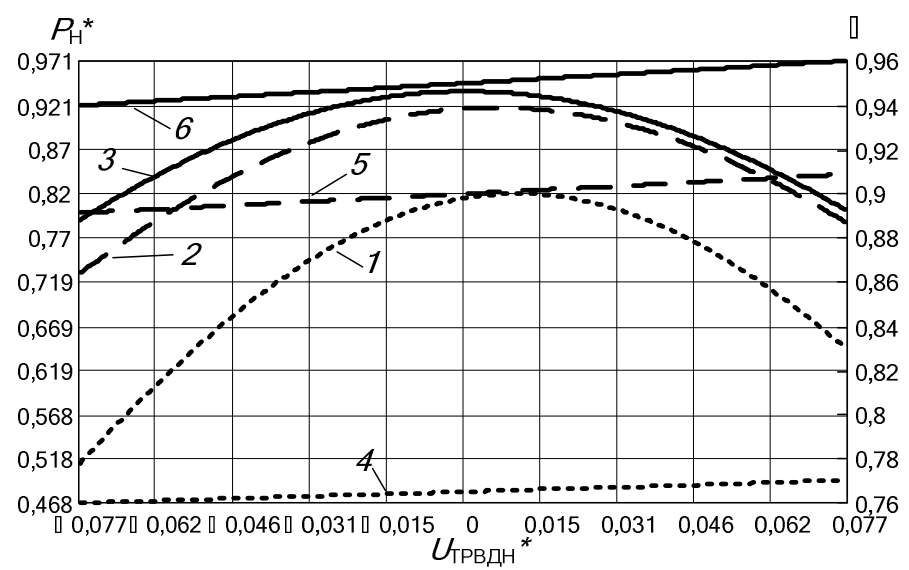

Рис. 8. Зависимости мощности нагрузки, КПД системы от продольной составляющей напряжения ТРВДН: $1-\eta$ при $\varphi=60^{\circ}, 2-\eta$ при $\varphi=30^{\circ}, 3-\eta$ при $\varphi=0^{\circ}$, $4-P$ при $\varphi=60^{\circ}, 5-P$ при $\varphi=30^{\circ}, 6-P$ при $\varphi=0^{\circ}$ 
В результате проведенных исследований установлено, что включение ТРВДН позволяет загрузить линии пропорционально их пропускной способности в широком диапазоне изменения параметров параллельных ЛЭП. При этом за счет увеличения пропускной способности линий мощность нагрузки можно увеличить на 35 \%. Максимальное значения КПД при активной нагрузке $\left.\left(\varphi=0^{\circ} ; l_{1} / l_{2}=0,2 ; \varepsilon_{1} / \varepsilon_{2}=1,32\right) ; \eta=0,97\right)$, при активно-индуктивной нагрузке ( $\left.\varphi=30^{\circ} ; l_{1} / l_{2}=0,2 ; \varepsilon_{1} / \varepsilon_{2}=1,32\right) \eta=0,965$, при угле нагрузки $\varphi=60^{\circ}$ КПД составляет $\eta=0,943$.

Выводы. Разработанные имитационные и математические модели радиальной РЭС с параллельно работающими ЛЭП позволили получить области эффективной работы ТРВДН при различных режимах регулирования напряжения. Определены зависимости КПД линии электропередач, потерь мощности. Установлены зависимости токовой нагрузки параллельных линий от величины напряжения регулирования ТРВДН. Установлено, что в случае поперечного регулирования при изменении угла сдвига $\theta$ основной гармоники выходного напряжения ТРВДН на $\pm 5^{\circ}$ происходит изменение угла тока нагрузки в пределах $1^{\circ}$. Анализ характеристик показал, что использование разработанного устройства для управления потоками мощности повысит эффективность передачи электроэнергии потребителю.

Проведенные исследования доказали эффективность предлагаемых схемных решений ТРВДН. Внедрение такого устройства в РЭС позволит успешно управлять потоками мощностей и стабилизировать токи параллельно работающих ЛЭП.

Работа выполняется при финансовой поддержке Министерства науки и выстего образования РФ (соглашение № 14.577.21.0242 о предоставлении субсидии от 26.09.2017, уникальный идентификатор проекта RFMEFI57717X0242).

\section{Библиографический список}

1. Hadjsaid N., Cabonnadiere J. Cl. Smart grids: motivation, rates and perspective // Energetics abroad. - 2014. - Vol. 3. - P. 2-24.

2. Jalali, Aldeen M. Improving voltage stability margin using STATCOM-storage devices // IEEE PES Innovative Smart Grid Technologies Conference Europe. - 2016. DOI: 10.1109/ISGTEurope.2016.7856185 
3. Mohammad, Behnam M., Kazem Z., Mehdi A. Probabilistic allocation of thyristor-controlled phase shifting transformer for transient stability enhancement of electric power system // IETE Journal of Research. - 2015. - Vol. 61. - P. 382-391. DOI: 10.1080/03772063.2015.1023743

4. Балабанов М.С., Хамитов Р.Н Facts-устройства. Выбор при проектировании электрооборудования предприятий: монография. Омск: Изд-во ОмГТУ, 2015. - С. 183.

5. Kusko, Thompson M. Power Quality in Electrical Systems // McGraw Hill. - 2007. - P. 13-17.

6. Petintin J. O., Shaaban M. Voltage regulation in a smart distribution system incorporating variable renewable generation // 2014 IEEE Innovative Smart Grid Technologies - Asia (ISGT ASIA). - Kuala Lumpur. 20-23 May 2014. - P. 583-588. DOI: 10.1109/ISGT-Asia.2014.6873857

7. Применение фазоповоротных устройств с тиристорным управлением как элемента управляемых (гибких) линий электропередачи переменного тока / В.П. Жмуров, В.Н. Стельмаков, А.Н. Тарасов, Б.И. Гринштейн // Электротехника. - 2014. - № 1. - С. 2-11.

8. Асташев М.Г., Панфилов Д.И. Фазоповоротные устройства с тиристорными коммутаторами для активно-адаптивных электрических сетей // Электричество. - 2013. - № 8. - С. 60-64.

9. Стельмаков В.Н., Жмуров В.П., Тарасов А.Н., Фазоповоротные устройства с тиристорным управлением // Электротехника. - 2014. № 1. - С. 11-18.

10. Разработка автоматического регулятора напряжения для снижения электрических потерь и эффективного управления потоками мощности в распределительных электрических сетях / Е.Н. Соснина, А.А. Асабин [и др.] // Соглашение с Мин-вом образов. и науки РФ № 14.577.21.0242 о предоставлении субсидии от 26.09.2017, уникальный идентификатор проекта RFMEFI57717X0242. - 2017. - T. 1.

11. Power flow control device prototype tests / E. Sosnina, A. Loskutov, A. Asabin, R. Bedretdinov, E. Kryukov // IEEE PES Innovative Smart Grid Technologies Conference Asia. - 2016. - P. 312-316. DOI: 10.1109/ISGT-Asia.2016.7796404

12. Тиристорное фазоповоротное устройство с вольтодобавочным трансформатором для сети среднего напряжения / Е.Н. Соснина, А.А. Асабин, А.А. Кралин, Е.В. Крюков // Роспатент. - 2016. № 2621062. 
13. Sharma A., Jaswal R.A. Congestion management by TCPST // IEEE 9 Bus System Using Matlab Simulink. International Journal of Engineering Sciences \& Research Technology. - 2013. - Vol. 2. - № 8. P. 1959-1963.

14. Солнцев Е.Б., Петрицкий С.А., Юртаев С.Н. Разработка имитационной модели тиристорного регулятора напряжения // Интеллектуальная электротехника. - 2018. - № 4. - С. 90-99.

15. Солнцев Е.Б., Петрицкий С.А., Юртаев С.Н. Расчетноаналитическая модель участка распределительной электрической сети 6-20 кВ с тиристорным регулятором напряжения // Интеллектуальная электротехника. - 2018. - № 2. - С. 39-52.

16. Асабин А.А., Кралин А.А. Энергетические показатели тиристорного регулятора переменного напряжения с вольтодобавочным трансформатором при поочередном двухзонном управлении // Интеллектуальная электротехника. - 2018. - № 2. - С. 93-104.

17. Research on power flow algorithm of power system with UPFC / J. Wen, Y. Tang, C. Xie, X. Chen, Y. Wang, N. An // 2018 International Conference on Power System Technology, POWERCON 2018 - Proceedings. - 2018. - P. 2453-2457.

18. Shahgholian G., Mahdavian M., Janghorbani M., Eshaghpour I., Ganji E. Analysis and simulation of UPFC in electrical power system for power flow control // ECTI-CON 2017 14th International Conference on Electrical Engineering/Electronics, Computer, Telecommunications and Information Technology. - 2017. - P. 62-65. DOI: 10.1109/ECTICon.2017.8096173

19. UPFC-based economic operation of distribution network / X. Ge, R. Wei, K. Zhou, F. Gao // Power System Technology. - 2018. Vol. 32. - P. 2585-2593. DOI: 10.13335/j.1000-3673.pst.2017.3008

20. ПУЭ 7 - Правила устройства электроустановок, 2013 // Доступ из справочно-правовой системы КонсультантПлюс.

\section{References}

1. Hadjsaid N., Cabonnadiere J. Cl. Smart grids: motivation, rates and perspective. Energetics abroad, 2014, vol. 3, pp. 2-24.

2. Jalali, Aldeen M. Improving voltage stability margin using STATCOM-storage devices. IEEE PES Innovative Smart Grid Technologies Conference Europe, 2016. DOI: 10.1109/ISGTEurope.2016.7856185 
3. Mohammad, Behnam M., Kazem Z., Mehdi A. Probabilistic allocation of thyristor-controlled phase shifting transformer for transient stability enhancement of electric power system. IETE Journal of Research, 2015, vol. 61, pp. 382-391. DOI: 10.1080/03772063.2015.1023743

4. Balabanov M.S., Khamitov R.N. Facts-ustroistva. Vybor pri proektirovanii elektrooborudovaniia predpriiatii [FACTS-devices. Selection by industrial electrical equipment design]. Omsk: Omskii gosudarstvennyi tekhnicheskii universitet, 2015. $183 \mathrm{p}$.

5. Kusko, Thompson M. Power quality in electrical systems. McGraw Hill, 2007, pp. 13-17.

6. Petintin J. O., Shaaban M. Voltage regulation in a smart distribution system incorporating variable renewable generation. 2014 IEEE Innovative Smart Grid Technologies - Asia (ISGT ASIA). Kuala Lumpur, 20-23 May 2014, pp. 583-588. DOI: 10.1109/ISGT-Asia.2014.6873857

7. Zhmurov V.P., Stel'makov V.N., Tarasov A.N., Grinshtein B.I. Primenenie fazopovorotnykh ustroistv $\mathrm{s}$ tiristornym upravleniem kak elementa upravliaemykh (gibkikh) linii elektroperedachi peremennogo toka [Application of thyristor-controlled phase-shifting arrangements as an element of controllable (flexible) alternating current power lines]. Elektrotekhnika, 2014, no. 1, pp. 2-11.

8. Astashev M.G., Panfilov D.I. Fazopovorotnye ustroistva s tiristornymi kommutatorami dlia aktivno-adaptivnykh elektricheskikh setei [Phase-shifting devices with thyristor switches for active-adaptive electric networks]. Elektrichestvo, 2013, no. 8, pp. 60-64.

9. Stel'makov V.N., Zhmurov V.P., Tarasov A.N., Fazopovorotnye ustroistva $\mathrm{s}$ tiristornym upravleniem [Thyristor-controlled phase-shifting devices]. Elektrotekhnika, 2014, no. 1, pp. 11-18.

10. Sosnina E.N., Asabin A.A. et al. Razrabotka avtomaticheskogo reguliatora napriazheniia dlia snizheniia elektricheskikh poter' i effektivnogo upravleniia potokami moshchnosti $\mathrm{v}$ raspredelitel'nykh elektricheskikh setiakh [Development of automatic voltage regulator to decrease electrical losses and power flows efficient control in electrical distribution networks]. Soglashenie s Ministerstvom obrazovaniia i nauki RF № 14.577.21.0242 o predostavlenii subsidii ot 26.09.2017, unikal'nyi identifikator proekta RFMEFI57717X0242, 2017, vol. 1.

11. Sosnina E., Loskutov A., Asabin A., Bedretdinov R., Kryukov E. Power flow control device prototype tests. IEEE PES Innovative Smart 
Grid Technologies Conference Asia, 2016, pp. 312-316. DOI: 10.1109/ISGT-Asia.2016.7796404

12. Sosnina E.N., Asabin A.A., Kralin A.A., Kriukov E.V. Tiristornoe fazopovorotnoe ustroistvo $\mathrm{s}$ vol'todobavochnym transformatorom dlia seti srednego napriazheniia [Thyristor phase shifting device with booster transformer for medium voltage networks]. Rospatent, 2016, no. 2621062.

13. Sharma A., Jaswal R.A. Congestion management by TCPST. IEEE 9 Bus System Using Matlab Simulink. International Journal of Engineering Sciences \& Research Technology, 2013, vol. 2, no. 8, pp. 1959-1963.

14. Solntsev E.B., Petritskii S.A., Iurtaev S.N. Razrabotka imitatsionnoi modeli tiristornogo reguliatora napriazheniia [Development of simulation model of thyristor voltage regulator]. Intellektual'naia elektrotekhnika, 2018, no. 4, pp. 90-99.

15. Solntsev E.B., Petritskii S.A., Iurtaev S.N. Raschetnoanaliticheskaia model' uchastka raspredelitel'noi elektricheskoi seti 6-20 kV $\mathrm{s}$ tiristornym reguliatorom napriazheniia [Design and analytical model of 6-20 kV distribution electric network section with thyristor voltage regulator]. Intellektual'naia elektrotekhnika, 2018, no. 2, pp. 39-52.

16. Asabin A.A., Kralin A.A. Energeticheskie pokazateli tiristornogo reguliatora peremennogo napriazheniia s vol'todobavochnym transformatorom pri poocherednom dvukhzonnom upravlenii [Energy indicators of thyristor ac voltage regulator with booster transformer under alternate two-zone control]. Intellektual'naia elektrotekhnika, 2018, no. 2, pp. 93-104.

17. Wen J., Tang Y., Xie C., Chen X., Wang Y., An N. Research on power flow algorithm of power system with UPFC. 2018 International Conference on Power System Technology, POWERCON 2018 - Proceedings, 2018, pp. 2453-2457.

18. Shahgholian G., Mahdavian M., Janghorbani M., Eshaghpour I., Ganji E. Analysis and simulation of UPFC in electrical power system for power flow control. ECTI-CON 2017 14th International Conference on Electrical Engineering/Electronics, Computer, Telecommunications and Information Technology, 2017, pp. 62-65. DOI: 10.1109/ECTICon.2017.8096173

19. Ge X., Wei R., Zhou K., Gao F. UPFC-Based Economic Operation of Distribution Network. Power System Technology, 2018, vol. 32, pp. 2585-2593. DOI: 10.13335/j.1000-3673.pst.2017.3008

20. PUE 7 - Pravila ustroistva elektroustanovok [Electric Install Code], 2013. 


\section{Сведения об авторах}

Кралин Алексей Александрович (Нижний Новгород, Россия) кандидат технических наук, доцент, заведующий кафедрой «Теоретическая и общая электротехника» Нижегородского государственного технического университета им. Р.Е. Алексеева (603950, Нижний Новгород, ул. Минина, 24, e-mail: akralin@yandex.ru).

Крюков Евгений Валерьевич (Нижний Новгород, Россия) кандидат технических наук, старший преподаватель кафедры «Электроэнергетика, электроснабжение и силовая электроника» Нижегородского государственного технического университета им. Р.Е. Алексеева (603950, Нижний Новгород, ул. Минина, 24, e-mail: kryukov@nntu.ru).

Еременко Владимир Викторович (Нижний Новгород, Россия) инженер кафедры «Электроэнергетика, электроснабжение и силовая электроника» Нижегородского государственного технического университета им. Р.Е. Алексеева (603950, Нижний Новгород, ул. Минина, 24, e-mail: eryomenko.vladimir96@mail.ru).

\section{About the authors}

Kralin Alexey Aleksandrovich (Nizhny Novgorod, Russian Federation) is a Ph.D. in Technical Sciences, Associate Professor, head of the chair "Theoretical and General electrical engineering" Nizhny Novgorod State Technical University named after R.E. Alekseeva (603950, Nizhny Novgorod, 24, Minin str., e-mail: akralin@yandex.ru).

Kryukov Evgeny Valerevich (Nizhny Novgorod, Russian Federation) is a Ph.D. in Technical Sciences, Assistant Professor of the chair "Electrical power supply and power electronics" Nizhny Novgorod State Technical University named after R.E. Alekseeva (603950, Nizhny Novgorod, 24, Minin str., e-mail: kryukov@nntu.ru).

Eremenko Vladimir Viktorovich (Nizhny Novgorod, Russian Federation) is e Engineer of the chair "Electrical power supply and power electronics" Nizhny Novgorod State Technical University named after R.E. Alekseeva (603950, Nizhny Novgorod, 24, Minin str., e-mail: eryomenko.vladimir96@mail.ru).

Получено 17.07.2019 